\title{
Training effect of exchange-bias bilayers within the domain state model
}

\author{
A. G. Biternas, ${ }^{1, *}$ U. Nowak, ${ }^{2}$ and R. W. Chantrell ${ }^{1}$ \\ 'Department of Physics, The Computational Magnetism Group, University of York, York YO10 5DD, United Kingdom \\ ${ }^{2}$ Fachbereich Physik, Universität Konstanz, D-78457 Konstanz, Germany
}

\begin{abstract}
An investigation of the temperature dependence of the training effect of various exchange coupled bilayers with different types of anisotropy is presented. We use an atomistic model for the magnetic interactions within a classical Heisenberg spin Hamiltonian. In general, the behavior of the exchange-bias field is separated into low- and high-temperature regions. This separation is made according to the trend of exchange-bias field after the second hysteresis loop and the parameters of the power-law fit for these fields. It is found that with increasing antiferromagnetic thickness, systems follow the same temperature trend but with lower values of the exchange-bias field and a weaker training effect. This is due to the fact that thicker antiferromagnetic layers lead to increased stability of the antiferromagnetic domains. Also, the behavior of the coercive fields is investigated, concluding that the training effect occurs predominantly in the first half of the hysteresis loop.
\end{abstract}

PACS number(s): $75.70 . \mathrm{Cn}, 75.60 . \mathrm{Jk}, 75.40 . \mathrm{Mg}$

\section{INTRODUCTION}

Exchange bias (EB) is the phenomenon in which the hysteresis loop of a system, usually comprising of two coupled magnetic materials with different magnetic orders, is shifted along the magnetic field axis. The most common EB system is a bilayer consisting of a ferromagnet (FM) in contact with an antiferromagnet (AFM). This unidirectional magnetic anisotropy is induced after cooling the system below the blocking temperature $T_{B}$ of the system while, above this temperature, the shift ceases to exist. Furthermore, EB is often accompanied by an enhancement of coercivity and by a gradual degradation of the EB field during consecutive hysteresis loops. The latter is known as the training effect, which is often observed in connection with a change in shape of the hysteresis loop in addition to the reduction in the EB field and coercivity. However, according to Hoffmann, ${ }^{1}$ some systems do not exhibit training effects. Although EB systems are being used extensively in giant magnetoresistance - or tunneling magnetoresistance-based spintronic devices, ${ }^{2-4}$ a full microscopic theory that explains the huge range of experimental results is still not established.

In 1956 Meiklejohn and Bean ${ }^{5}$ observed EB for the first time in fine $\mathrm{Co} / \mathrm{CoO}$ particles after an initial field cooling process. They suggested an intuitive model ${ }^{6}$ with an exchange coupling between the FM and an uncompensated AFM interface, which resulted in an EB field two orders of magnitude larger than the experimental ones. Subsequently Néel ${ }^{7}$ and Mauri et $a l^{8}$ considered a domain wall created in the AFM parallel to the interface, during field reversal. Later many other models considered this type of mechanism, for example, Koon ${ }^{9}$ generalized this mechanism for the case of compensated interfaces, with the spins restricted to the interface plane. However, it was shown later by Schulthess and Butler $^{10}$ that if in Koon's model the spins are not restricted to an $x-y$ plane the EB vanishes. On the other hand, Malozemoff's model ${ }^{11-13}$ used domain walls that are perpendicular to the plane of the AFM interface. These domain walls are favored by a roughness of the interface between AFM and FM. Numerical investigations ${ }^{14}$ of $\mathrm{EB}$ dependence on the interface roughness were shown to coincide with Malozemoff's model for minimal bulk dilution and interface roughness.

In 2000 , a new model ${ }^{15}$ was introduced considering domains in the AFM created during the initial cooling process due to a dilution of the AFM with nonmagnetic defects. The resulting shift of the hysteresis loop was attributed to the exchange interaction of the FM with the irreversible magnetization of the AFM domains. The domain walls are pinned at the defects causing the metastability of the domains. In the framework of the so-called domain state model a range of phenomena has been investigated, such as the dependence of EB on the AFM thickness, ${ }^{16}$ the dilution, ${ }^{17}$ the temperature, and also the training effect. ${ }^{18}$ The validity of the domain state model is supported by experimental results ${ }^{19-21}$ as well as by direct spectroscopic observations. ${ }^{22,23}$

The training effect was first discovered by Paccard et al. ${ }^{24}$ in $\mathrm{Co}-\mathrm{CoO}$ and $\mathrm{NiFe}-\mathrm{AFM}$ systems. Training effects are present in heterosystems containing polycrystalline AFM layers $^{25-29}$ as well as in systems with a single crystalline AFM (Ref. 3) (with a reduced effect) and in all ferrimagnetic bilayers. ${ }^{30}$ Néel $^{7}$ claimed that the training effect in EB systems is an effect similar to training effect in a FM. He assumed that the phenomenon is related to the negative coupling between FM domains, which will be caused by an atomic step on the AFM interface.

As shown by Hoffmann's 1 model and supported by many experimental results, the training effect can be divided to thermal and athermal effects. This distinction was introduced first by Fernandez-Outon et $a .^{31}$ and is based on the behavior of the first loop EB field. The athermal training is considered to be the large shift of the descending branch of the first loop, which persists even at very low temperatures where the thermal training, driven by thermally activated magnetization processes, ceases to exist.

The initial cooling procedure produces an AFM spin structure which may be in a metastable state; the training effect can then be considered to be the macroscopic signature of the configurational rearrangement of the spin structure toward equilibrium. Despite that, because of the variety of experimental results, the uniqueness of the mechanism has to 
be questioned. These reconfigurations could be a reorientation of the AFM domains at the AFM/FM interface during field reversal or domain wall movements. Certainly, the AFM domain state of three-dimensional systems is metastable, giving rise to nonequilibrium phenomena and, consequently, to training effects.

In the present paper we will focus on the behavior of the FM for different EB systems using Monte Carlo simulations of the domain state model. We will investigate systems with uniaxial and biaxial anisotropies and different AFM thicknesses. The present paper is organized as follows. In the next section we give a brief description of the model used, including especially the terms for uniaxial and biaxial anisotropies. In Sec. III, our results are discussed in two subsections. The first one is on the hysteresis loop and EB field behavior, introducing the power-law fit for further analysis. The second subsection discusses the behavior the coercive fields.

\section{MODEL}

The model which is used in the following is a classical Heisenberg model for a FM monolayer exchange coupled to a diluted AFM (two or five monolayers). The overall system is a multilayered film of lateral size of $128 \times 128$ atoms for all the cases of this study. The lateral system size was varied in order to check that the results are not subjected to finitesize effects. This size used guarantees that an AFM domain structure fits into the system. Simple cubic crystal packing is used with periodic boundary conditions in the lateral direction and open boundary conditions in the out-of-plane direction.

FM and AFM are modeled as classical Heisenberg spins coupled with nearest-neighbor exchange constants $J_{\mathrm{FM}}$ and $J_{\mathrm{AFM}}=-J_{\mathrm{FM}} / 2$. These exchange constants determine the $\mathrm{Cu}-$ rie temperature of the FM and the Neel temperature of the AFM, respectively. To obtain a well-defined hysteresis loop, a magnetocrystalline anisotropy is introduced in the FM. Its easy axis is the $x$ axis with anisotropy constant $d_{x}=0.1 J_{\mathrm{FM}}$, which sets the zero-temperature limit for the coercive fields for magnetization reversal by coherent rotation (StonerWohlfarth limit). In addition the dipolar interaction is approximated by an additional uniaxial easy-plane anisotropy ( $z$ axis as hard axis with anisotropy constant $d_{z}=-0.1 J_{\mathrm{FM}}$ ), which includes the shape anisotropy of the film. However, its strength does not influence our results. For the AFM, two cases of magnetocrystalline anisotropy are considered: one with a single easy axis (uniaxial anisotropy) parallel to that of the FM and one with two easy axes (biaxial anisotropy) at $45^{\circ}$ to that of the FM.

Thus, the total Hamiltonian of our system is

$$
\mathcal{H}_{\mathrm{TOT}}=\mathcal{H}_{\mathrm{FM}}+\mathcal{H}_{\mathrm{AFM}}+\mathcal{H}_{\mathrm{INT}} \text {. }
$$

Here, $\mathcal{H}_{\mathrm{FM}}$ is the FM Heisenberg Hamiltonian given by

$$
\mathcal{H}_{\mathrm{FM}}=-J_{\mathrm{FM}} \sum_{\substack{\langle i, j\rangle \\ \in \mathrm{FM}}} \mathbf{S}_{i} \cdot \mathbf{S}_{j}-\sum_{i \in \mathrm{FM}}\left(d_{x} S_{i x}^{2}+d_{z} S_{i z}^{2}\right)-\sum_{i \in \mathrm{FM}} \mu \mathbf{B} \cdot \mathbf{S}_{i},
$$

where $\mathcal{H}_{\mathrm{AFM}}$ is the AFM classical Heisenberg Hamiltonian, which is given by

$$
\mathcal{H}_{\mathrm{AFM}}=-J_{\mathrm{AFM}} \sum_{\substack{\langle i, j\rangle\\}} \epsilon_{i} \epsilon_{j} \boldsymbol{\sigma}_{i} \cdot \sigma_{j}+E_{\mathrm{AnM}}-\sum_{i \in \mathrm{AFM}} \mu \mathbf{B} \cdot \epsilon_{i} \boldsymbol{\sigma}_{i},
$$

and $\mathcal{H}_{\mathrm{INT}}$ is the interaction Hamiltonian between FM and AFM, which is given by

$$
\mathcal{H}_{\mathrm{INT}}=-J_{\mathrm{INT}} \sum_{\substack{(i \in \mathrm{FM}, j \in \mathrm{AFM})}} \mathbf{S}_{i} \cdot \epsilon_{j} \boldsymbol{\sigma}_{j},
$$

where, for simplicity, $J_{\mathrm{INT}}$ is assumed to be equal to $\left|J_{\mathrm{AFM}}\right|$. The interaction between FM and AFM is considered only for the two interfacial layers, i.e., one of the AFMs and one of the FMs. Also, the interface is considered perfect without any roughness. The quantities $\mathbf{S}_{i}$ and $\boldsymbol{\sigma}_{i}$ are unit vectors describing atom spins with magnetic moment $\mu$. The magnetic field $\mathbf{B}$ is applied at an angle of $20^{\circ}$ to the $x$ axis and in the $x-y$ plane. This angle was chosen such that systems with uniaxial AFM anisotropy show training effect. For larger values of this angle we have no training effect independent of temperature. Due to the dilution with nonmagnetic impurities not all AFM lattice sites have an associated spin. This is introduced using the variable $\epsilon_{i}=0,1$, with individual values determined such that $p=N^{-1} \sum \epsilon_{i}$ is the packing fraction of the AFM spins.

The term $E_{\text {Anis }}$ describes the magnetocrystalline anisotropy energy of the AFM, which is given by either

$$
E_{\text {Anis }}=-\sum_{i \in \mathrm{AFM}} \epsilon_{i} k_{x} \sigma_{i x}^{2}
$$

or

$$
E_{\mathrm{Anis}}=-\sum_{i \in \mathrm{AFM}} \epsilon_{i}\left(k_{x} \sigma_{i x}^{4}+k_{y} \sigma_{i y}^{4}\right)
$$

for the case of uniaxial or biaxial symmetry, respectively. Equations (5) and (6) describe magnetocrystalline anisotropies with easy axes lying in the $x-y$ plane, with the $x$ axis being the easy axis of the uniaxial anisotropy and $x$ and $y$ axes being the easy axes of the biaxial anisotropy. $k_{x}$ and $k_{y}$ are the anisotropy constants, with $k_{x}=k_{y}=J_{\mathrm{FM}}$, which create high anisotropy in the AFM and subsequently generates small domain walls with width being equal to one atomic site. For the case of the biaxial anisotropy, the easy axes are rotated by $45^{\circ}$, hence to be bisected by the easy axis of the FM. This is due to our requirement of not to favor any particular direction because of the interface exchange interaction with the FM.

Monte Carlo simulation techniques are used to investigate the system implemented as heat-bath algorithm (for details, see Ref. 32). The AFM spin trial step consists of (i). a total flip of the spin or (ii) a small random movement constrained to a maximum angle of $22^{\circ}$. Both trial steps are chosen with equal probability. The trial step for the FM spins is only a small random movement constrained to a maximum angle of $42^{\circ}$. The choice of the aforementioned angles and procedure does not affect the results but is used to optimize the computational efficiency. ${ }^{32}$ 


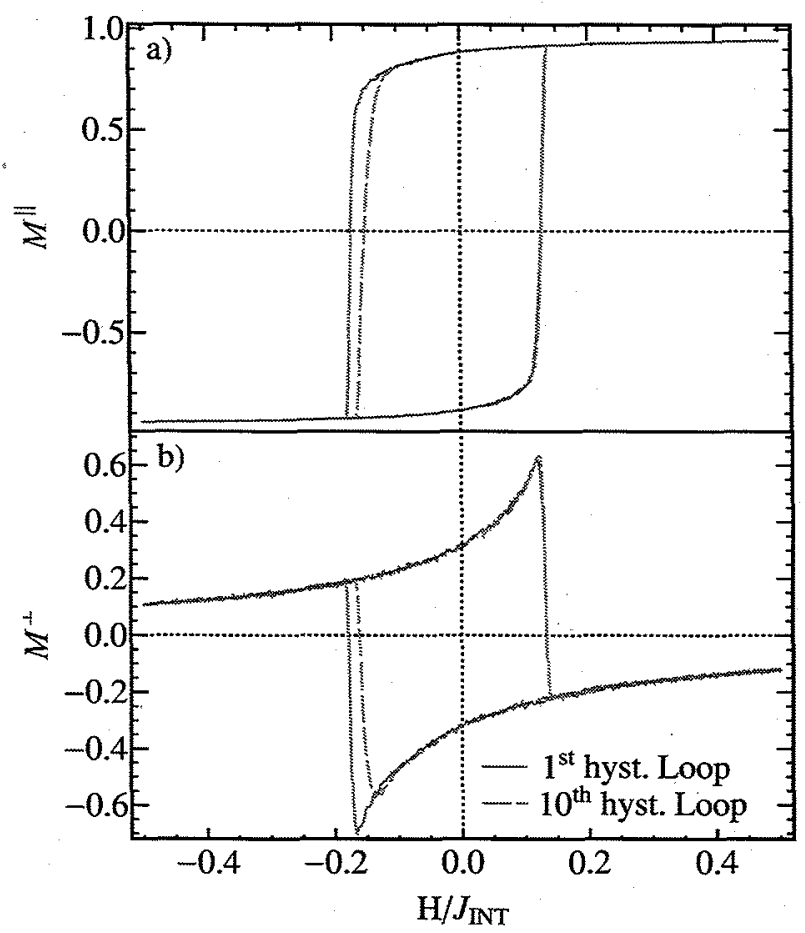

FIG. 1. (Color online) (a) Parallel and (b) transverse components of magnetization of the first (solid line) and of a subsequent (dashed line) hysteresis loop. The magnetization is normalized to its zero-temperature saturation value and the field is in units of $J_{\mathrm{INT}}$.

The Néel temperatures were calculated for each individual system separately using a heating process from almost zero temperature from the long-range ordered antiferromagnetic state. ${ }^{33}$ The inflection point of the staggered magnetization was then calculated as a function of temperature, estimating the Neel temperature for the uniaxial system to be $k_{B} T_{N}=0.93 J_{\mathrm{INT}}$ and $1.14 J_{\mathrm{INT}}$, and for the biaxial system $k_{B} T_{N}=0.60 J_{\mathrm{INT}}$ and $0.81 J_{\mathrm{INT}}$ for two and five layer AFM thicknesses, respectively. The cooling process was performed using $0.5 \times 10^{6}$ Monte Carlo steps, starting from $k_{B} T$ $=3.4 J_{\mathrm{INT}}$ down to the desired temperatures. Each hysteresis loop is calculated using the same number of Monte Carlo steps. For the investigation of the training effect, consecutive hysteresis loops were calculated using each time the state of the previous loop as the initial state. Also, we calculated statistical averages of the EB field and other quantities over 20 different realizations of random number sequences. The main effect of this for the simulations is the different realizations of defect distributions in the AFM.

\section{RESULTS}

\section{A. Exchange-bias field}

The atomistic model presented earlier has been applied to calculations of systems with uniaxial and biaxial anisotropies for the AFM as well as different AFM thicknesses. A typical hysteresis loop calculated solely from the FM part of the system is shown in Fig. 1, where the top part shows the parallel (II) component of magnetization and the bottom part shows the perpendicular $(1)$ component. The first and the tenth hysteresis loops are presented. The training effect in the parallel component appears as a movement of the left-hand branch of the hysteresis loop. The training effect in the perpendicular component is manifested as a decrease in the maximum of the left-hand magnetization during reversal.

These findings indicate a symmetrical first loop with a predominantly coherent rotation as the reversal mode. This gradually changes to less symmetrical loops with (only) the left-hand branch becoming less uniform. The symmetry of the hysteresis loop during reversal was investigated thoroughly by Beckmann et al..$^{34,35}$ and was shown to depend on the angle between the easy axis of the AFM or FM and the magnetic field. As was shown, even the first loop can be asymmetric for certain angles. Therefore, the transition from a symmetric to an asymmetric mode is not a general effect, but it occurs for the specific angle we used in the current investigations $\left(20^{\circ}\right)$. For other angles, the asymmetry can exist from the first hysteresis loop onward. The reason for this behavior can be found in the change in the mean effective field acting in the FM during reversal. ${ }^{34}$ This field has three different contributions, namely, from the exchange field of the AFM, the external magnetic field, and the anisotropy FM field. The EB is the one which changes during training, causing the mean effective field to change. For the angle we used in this paper, during training the mean effective field becomes more aligned with the FM magnetization, leading to a smaller torque on the FM magnetization. This consequently favors less uniform reversal modes.

The exchange-bias field $\left(H_{\mathrm{eb}}\right)$ and the coercivity $\left(H_{c}\right)$ were calculated for different temperatures for every configuration. We investigated four different system configurations: two with a uniaxial AFM anisotropy and two with a biaxial anisotropy, both with two and five AFM layers. The dependences of $H_{\mathrm{eb}}$ and $H_{c}$ on the hysteresis loop cycle number are presented Fig. 2 for different temperatures and AFM thicknesses for the case of uniaxial AFM anisotropy. The error bars indicate the statistical error due to averaging over a set of 20 different realizations of the defect distribution in the AFM.

It is obvious that for all kind of parameters the largest decrease in the EB field takes place between the first and the second hysteresis loops. The training effect becomes weaker for the subsequent hysteresis loops. The coercivity shows a qualitatively similar training effect as observed for the EB field. For temperatures as low as only $1 \%$ of the Néel temperature $\left(0.0075 J_{\text {int }}\right), H_{\mathrm{eb}}$ is reduced by about $10-15 \%$ and $H_{c}$ is reduced by about $1-2 \%$ after ten loops when compared with its initial value. This is in good agreement with experimental findings for epitaxial $\mathrm{NiFe} / \mathrm{FeMn} \mathrm{EB}$ bilayers $^{36}$ where for a temperature of $5 \mathrm{~K}$ a corresponding decrease in $H_{\text {eb }}$ of about $30-35 \%$ was found and 2-4\% for $H_{c}$. This suggests that there is a domain state in the bilayer which may arise from the interface roughness or nonmagnetic defects in the epitaxial film.

For a better understanding of the training effect and for further analysis of the results, a fitting procedure was applied. The common empirical power law connecting $H_{\mathrm{eb}}$ and the number of consecutive loops is given by 


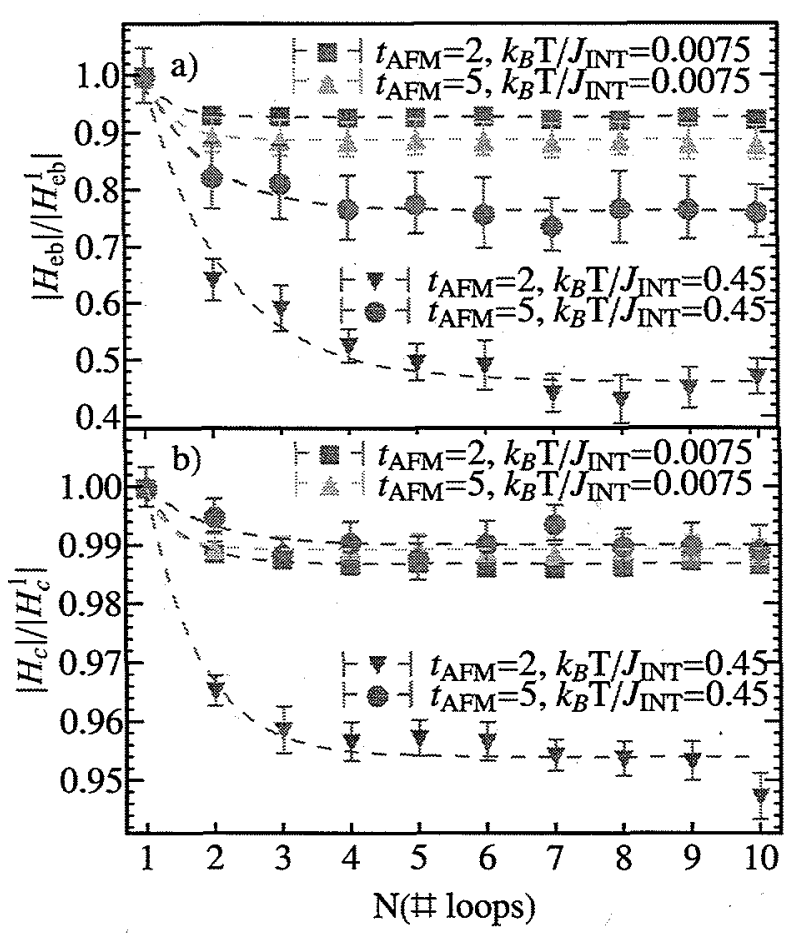

FIG. 2. (Color online) Training effect of (a) the exchange-bias field and (b) the coercivity for different temperatures and AFM thicknesses. AFM with uniaxial anisotropy.

$$
H_{\mathrm{eb}}(N)=H_{\mathrm{eb}}^{e}+k / \sqrt{N},
$$

where $k$ is a constant and $H_{\mathrm{eb}}^{e}$ is the equilibrium exchange bias, i.e., the remaining $H_{\mathrm{eb}}$ after an infinite number of loops. Introduced first by Paccard et al., ${ }^{24}$ this law holds only for $(N>1)$ and, consequently, cannot describe the steep training effect between the first and the second loops. Therefore, we additionally analyze the difference $d H_{\mathrm{eb}}$ of the power law as extrapolated to the first hysteresis loop and the actual simulation result for exchange-bias field of the first loop,

$$
d H_{\mathrm{eb}}=H_{\mathrm{eb}}^{1}-\left(H_{\mathrm{eb}}^{e}+k\right) .
$$

For clarity, we used the normalized quantities $k=(k$ $\left.\times 10^{-3}\right) / J_{\text {INT }}$ and $\Delta H_{\mathrm{eb}}=\left(d H_{\mathrm{eb}} \times 10^{-3}\right) / J_{\mathrm{INT}}$. However, the power law must be fitted to the original (not normalized) data. Some characteristic results are illustrated for three values of temperature in Fig. 3. Here, we show the original data along with the power-law fits and the resulting equilibrium EB field. First, even if we use the power law from the first loop onward, the fits only work well for $N>1$; for $N=1$ the fitting fails. As we can see for the lowest temperature the training effect is small, and the EB field seems to remain stable after only a small number of consecutive hysteresis loops. The first hysteresis loop deviates from the power law, a characteristic of athermal training. For higher temperatures the training effect is large, with the EB field decaying toward a much lower equilibrium value, but again with a bigger difference between the first loop and the power-law fit. Thus, this characteristic of the athermal training remains also in the

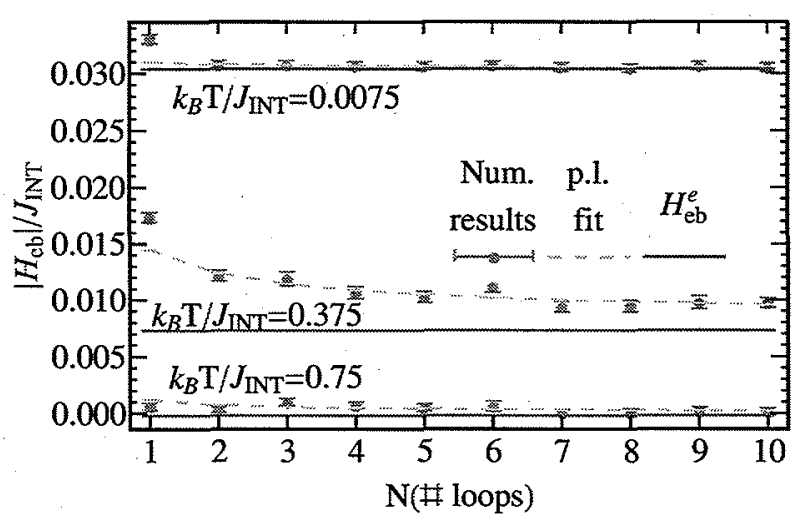

FIG. 3. (Color online) Numerical results (symbols) and power law (p.l.) fits (dashed lines) for three different temperatures along with the equilibrium exchange-bias fields (horizontal lines).

thermal training. Finally, for temperatures close to the blocking temperature the training effect is small, with the difference between the first loop and the power law also being small, a characteristic of low or zero training effect.

Our results for the temperature dependence of the training effect for the four aforementioned systems are shown in Fig. 4. The results for the first and second loops are shown as well as the equilibrium value obtained via the power-law fit. The fitting parameters $\kappa$ and the difference $\Delta H_{\text {eb }}$ are drawn in the upper graphs. The range of temperatures used is according to the system blocking temperature, which is defined as the temperature where the exchange-bias field becomes zero. Due to the rescaling of the temperature range with respect to the Néel temperature of each system, the real temperature range is different for each of the systems.

For the case of the AFM uniaxial anisotropy with a low AFM thickness [Fig. 4(a)], the strength of the training effect has a maximum for intermediate temperatures $\left(T / T_{N} \approx 0.4\right)$. This is because, at very low temperatures, the system is easily frozen in metastable states leading to a higher equilibrium EB field due to the lack of thermal excitation. By intermediate temperatures we mean temperatures in the center between zero and the blocking temperature of the system, i.e., the temperature where the EB field becomes zero. On the other hand for the highest temperatures the EB fields are rather small and drop quickly to the equilibrium value, which is equal to zero. In between is the temperature range with the highest training effect. This behavior is also apparent from the graph of the fitting parameter $\kappa$, which shows a maximum at $T / T_{N}=0.4$, while the difference $\Delta H_{\text {eb }}$ remains constant for not too high temperatures. Only for the highest temperatures close to the blocking temperature, $\Delta H_{\mathrm{eb}}$ decreases steeply. For the thicker AFM [Fig. 4(b)], the strength of the training effect follows the same trend but with the maximum of the training effect shifted toward higher temperatures. The main difference is that the additional AFM layers offer more stability to the system as well as to the created domains, so that the training effect is smaller.

The EB systems with a biaxial anisotropy of the AFM [Figs. 4(c) and 4(d)] show some significant differences in comparison to the uniaxial systems. First, the EB effect ap- 

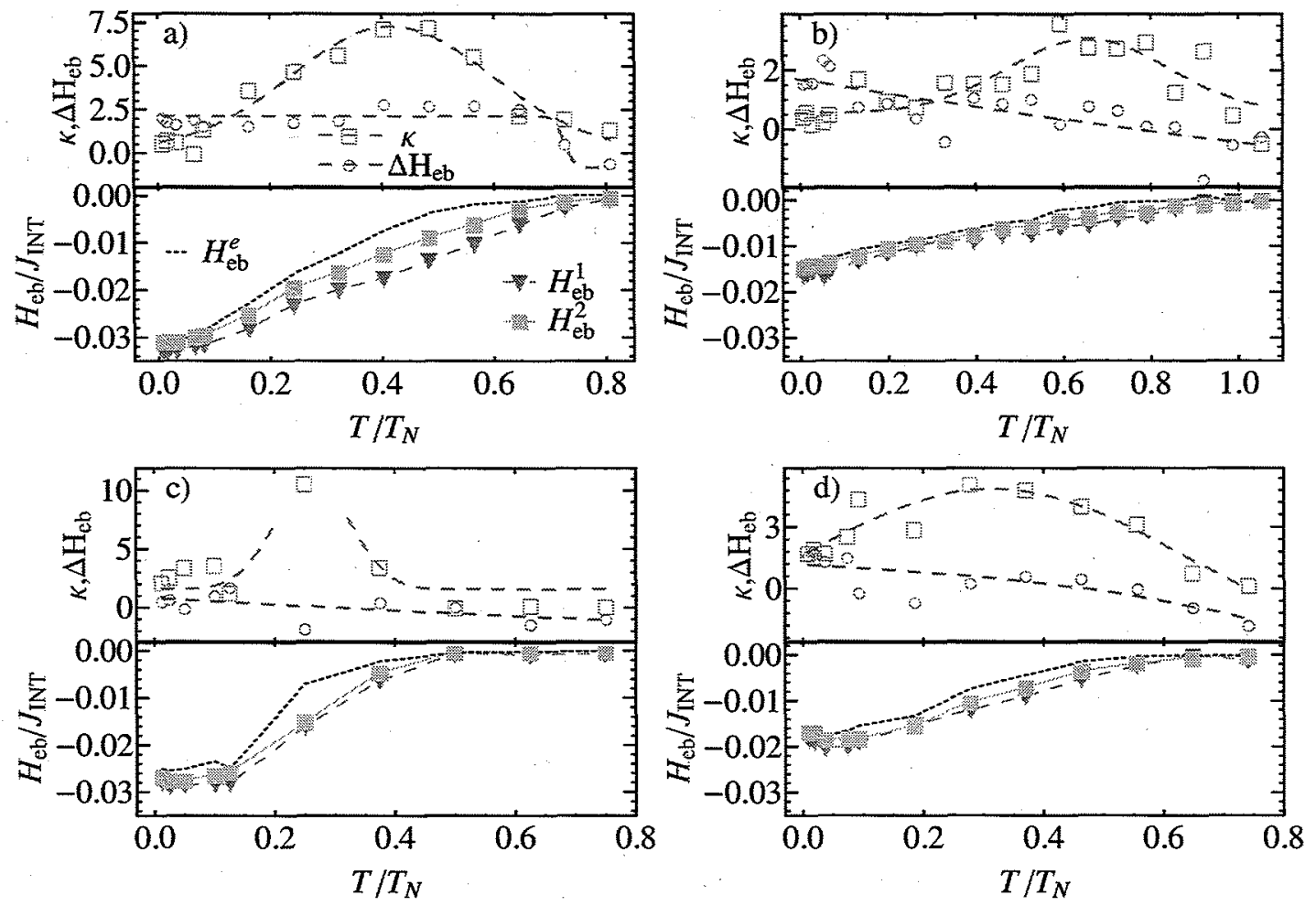

FIG. 4. (Color online) Temperature dependence of the training effect for systems with (a) uniaxial AFM anisotropy and $t_{\mathrm{AFM}}=2$, (b) uniaxial AFM anisotropy and $t_{\mathrm{AFM}}=5$, (c) biaxial AFM anisotropy and $t_{\mathrm{AFM}}=2$, and (d) biaxial AFM anisotropy and $t_{\mathrm{AFM}}=5$. The temperature axis is scaled to the Néel temperature of every system. $H_{\mathrm{eb}}^{1}$ and $H_{\mathrm{eb}}^{2}$ : EB field of the first and the second hysteresis loops, respectively. $H_{\mathrm{eb}}^{e}$ : equilibrium EB field following the power-law fit. Upper graphs: temperature dependence of the normalized fitting law parameter $\kappa$ and $\Delta H_{\mathrm{eb}}$ obtained as explained in the text.

pears only for lower temperatures. The resulting lower blocking temperature results from a lower-energy barrier for reversal of antiferromagnetic domains. As in the uniaxial case, the blocking temperature is also affected by the thickness of the AFM. For higher AFM thickness the blocking temperature increases, once again a result of the enhanced stability of the system due to the increased AFM thickness. Second, for the case of biaxial AFM anisotropy, the training effect shows a different dependence on temperature. This can be clearly seen from the graphs of the fitting parameters. The power-law parameter $\kappa$ shows a maximum at $T / T_{N}=0.3$ meaning that the training effect after the second loop is high. The difference $\Delta H_{\mathrm{eb}}$ has a monotonic decrease. In total, the difference between these two curves is in the lowtemperature range, where we see large training effect for the first to the second loop but much smaller for the subsequent loops. Similar effects exist for both of the thicknesses of the AFM; but, as previously, the EB persists at higher temperatures for the thicker AFM.

The behavior of the training effect can be compared with the experimental trend measured by Khapikov et $a l^{26}$ for a granular $\mathrm{NiO} / \mathrm{Ni}_{81} \mathrm{Fe}_{19}$ bilayer. If we consider that the blocking temperature of that system is around $250 \mathrm{~K}$, then the lower limit of temperature range that is used is around $T / T_{N}=0.16$ in terms of our temperature units for the system with the uniaxial anisotropy and a thickness equal to two layers. Thus, for this range, the temperature dependence is the same and the relative decrease in the EB field due to training is in very good agreement (both are $30 \%$ for $T / T_{N}$ $=0.16$ )

In general, the behavior of the EB field (where training exists), independent of the AFM thickness and anisotropy, can be separated in two regions according to the temperature:

(1) Athermal training. This is the region with low $\kappa$ and high $\Delta H_{\mathrm{eb}}$, usually in the low-temperature regime. As shown in Fig. 3, for $k_{B} T / J_{\mathrm{INT}}=0.0075$, the EB field of the first loop is significantly higher than the EB field of the subsequent loops. After the second loop the EB field remains constant.

(2) Thermal training. This is the region with high $\kappa$ and high $\Delta H_{\mathrm{eb}}$, usually in the higher-temperature regime, but not very close to the blocking temperature. As shown in Fig. 3, for $k_{B} T / J_{\mathrm{INT}}=0.375$, the $\mathrm{EB}$ field of the first loop is still much higher than those of the subsequent loops. But here, after the second loop the EB field is continuously decreasing with high parameter $\kappa$.

These two kinds of training effect are connected with the behavior of the antiferromagnet, particularly, with the increase in the stability of the AFM interface and the decrease in its magnetization. Our preliminary analysis suggests that the effect depends on the stability of the FM/AFM interface structure. More specifically, the interfacial spins of the AFM showed different behaviors during cycling. The largest portion was frozen toward the easy axes of the AFM anisotropy creating domains, and a smaller portion-which is in the 

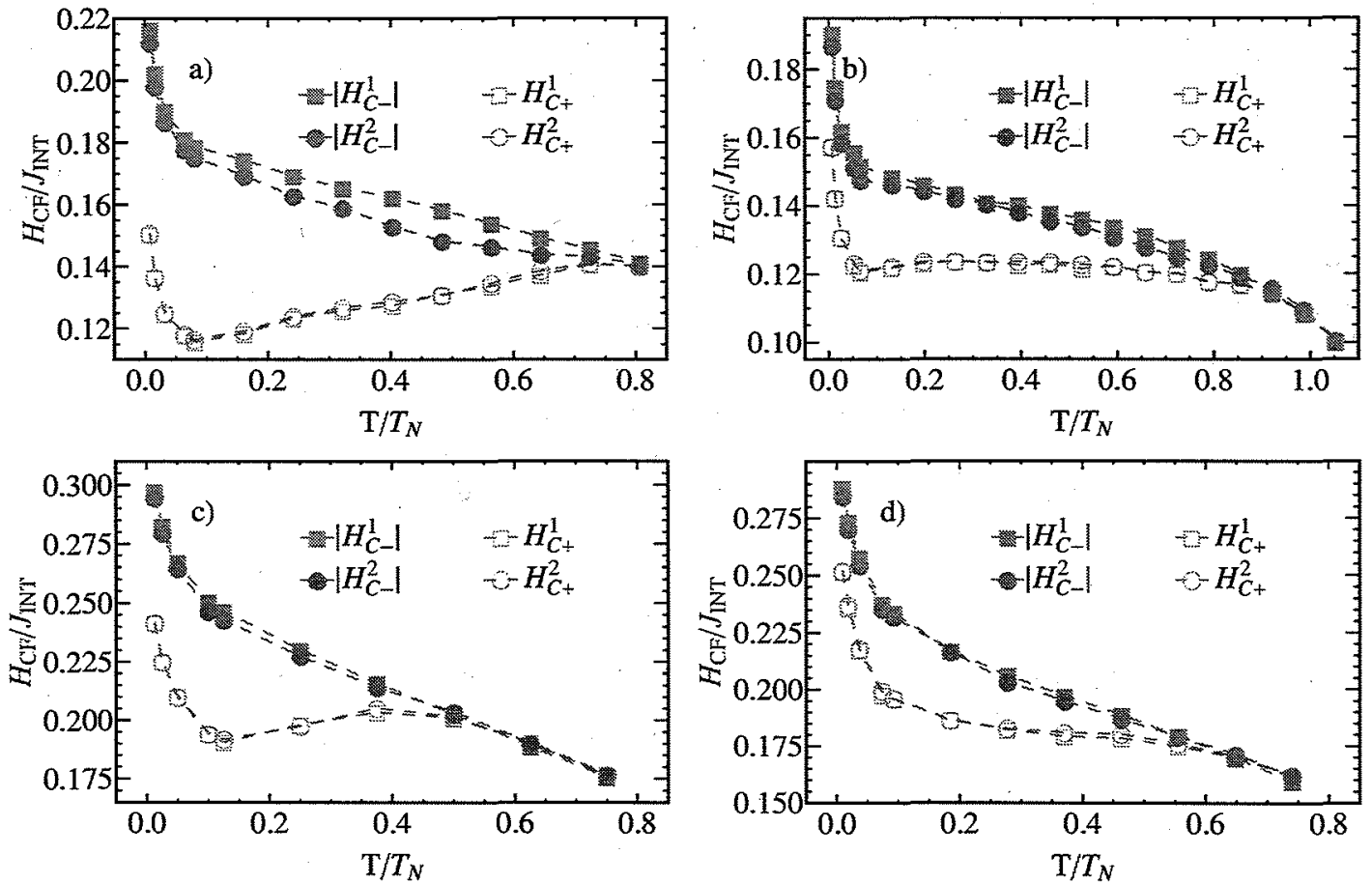

FIG. 5. (Color online) Temperature dependence of the training effect of the coercive fields for systems with (a) uniaxial AFM anisotropy and $t_{\mathrm{AFM}}=2$, (b) uniaxial $\mathrm{AFM}$ anisotropy and $t_{\mathrm{AFM}}=5$, (c) biaxial $\mathrm{AFM}$ anisotropy and $t_{\mathrm{AFM}}=2$, and (d) biaxial AFM anisotropy and $t_{\mathrm{AFM}}=5 . H_{C_{+}}^{1}$ and $H_{C_{+}}^{2}$ : positive coercive fields of the first and the second hysteresis loops, respectively, similarly for the absolute value of the negative coercive field. The temperature axis is scaled to the Néel temperature of every system and, as previously, the temperature range was adjusted according to the system blocking temperature (similar to Fig. 4).

domain walls-rotates with the external field causing the domain wall motion. The training effect is connected with the behavior of these spins which become frozen in a specific direction and consequently increasing the surface of the domains. This analysis is beyond the scope of the current paper and will be published separately.

A similar analysis was made by Radu and Zabel, ${ }^{37}$ where they showed that the training effect is connected with both interfacial and bulk spins which move toward equilibrium with different rates. Also, Roy et al. ${ }^{38}$ and Fitzsimmons et $a l .{ }^{39}$ analyzed the pinned and the unpinned spins not only on the interface but also in the bulk, characterizing them as compensated and uncompensated. They proposed a model using the results of their analysis that is consistent with our model and results. Finally, Morales et al. ${ }^{40}$ using a trilayer to eliminate the AFM/FM interlayer exchange coupling, showed that EB is not a purely interfacial effect and the bulk AFM spins are important for the pinning of the interfacial spins.

\section{B. Coercive fields}

Because of the possible asymmetry of the hysteresis loops, we also investigated the coercive fields of every cycle. The positive and the negative coercive fields of the first and second hysteresis loops are shown in Fig. 5 for each of the aforementioned systems. For simplicity the absolute value of the negative coercive field is used. When the curves of the positive and negative fields coincide, the EB field vanishes.

First, for all cases we can conclude that the training effect occurs predominantly in the negative coercive fields since there is hardly any difference in the subsequent curves for the positive coercive fields. This means also that one finds a decrease in coercivity during cycling over several loops. This is caused because during the initial cooling procedure an AFM domain state is formed with a spin structure optimized for the positive field and FM magnetization direction. Obviously, this domain structure is less stable when the field and the FM are reversed. Hence, more changes in the domain structure may occur on the opposite side of the loop as compared to the initial side where this structure is stable. Also, although nonmonotonically, the negative coercive field always decreases with increasing temperature. On the other hand, the positive coercive field has regional extrema. For the case of uniaxial AFM anisotropy, the positive coercive field has a minimum at rather low temperatures, $T / T_{N}$ $=0.08$.

The asymmetry in the hysteresis loop is suggested to be an effect of increasing population of the stable antiferromagnetic spins. More specifically, the irreversible magnetization of the antiferromagnetic interface decreases. This is caused by the increase in the stable spin population, which results in a decrease in their net magnetization, during consecutive field cycling. Thus, the unstable spin population in the AFM - which is responsible for the coercivity-reduces, causing the aforementioned behavior of coercivity. For lower 
temperatures, the behavior of the coercive field changes rapidly. Experimentally ${ }^{41}$ it was shown that the origin of the striking difference between the first magnetization reversal and all subsequent reversals is an effect of a metastable single domain state destroyed during the first reversal and transformed into a stable multidomain state.

\section{CONCLUSIONS}

In this work we have investigated the training effect of FM/AFM bilayers using Monte Carlo simulation of a domain state model for exchange bias. For the AFM uniaxial and biaxial anisotropies were considered as well as different thicknesses. For a deeper understanding a power-law fit was used to characterize the decay of the EB fields. Independent of the AFM thickness and anisotropy, the training effects can be separated in two regions according to the temperature: the athermal training region at low temperatures and the thermal training region for higher temperatures. Systems with thicker AFMs follow the same temperatune trend but with lower values of the EB field and a weaker training effect. This is due to the fact that the extra AFM layers offer more stability to the system as well as to the created domains.
Interestingly, the model of Hoffmann ${ }^{1}$ predicts no training effect for uniaxial materials. However, the calculations presented here show a significant training effect for uniaxial systems. We believe that this is associated with the detail of the interfacial spin configuration, which is not included in Hoffmann's model.

For all the systems, it is concluded that the training effect comes mainly from a shift of the negative coercive field. The asymmetry in the hysteresis loop is suggested to be an effect of an increase in the population of the stable antiferromagnetic spins. The EB field and coercivity are related with the stable and unstable AFM interface spins, respectively. The change in their population, an increase in the stable spins, and a decrease in the unstable spins make the two quantities correlated with each other. Finally, we showed that the blocking temperature for systems with uniaxial AFM anisotropy and larger thickness is higher.

\section{ACKNOWLEDGMENTS}

A.G.B. acknowledges financial support received by EU NEXBIAS Research Training Network (Contract No. HPRN-CT-2002-00296). Also, the White Rose Grid Computational Resources at the University of York is gratefully acknowledged.

\footnotetext{
*ab543@york.ac.uk

${ }^{1}$ A. Hoffmann, Phys. Rev. Lett. 93, 097203 (2004).

${ }^{2}$ J. Nogués, J. Sort, V. Langlais, V. Skumryev, S. Suriñach, J. S.

Muñoz, and M. D. Baró, Phys. Rep. 422, 65 (2005).

${ }^{3}$ J. Nogués and I. K. Schuller, J. Magn. Magn. Mater. 192, 203 (1999).

${ }^{4}$ R. L. Stamps, J. Phys. D 33, R247 (2000).

${ }^{5}$ W. H. Meiklejohn and C. P. Bean, Phys. Rev. 102, 1413 (1956).

${ }^{6}$ W. H. Meiklejohn and C. P. Bean, Phys. Rev. 105, 904 (1957).

${ }^{7}$ L. Néel, Ann. Phys. (Paris) 2, 61 (1967).

${ }^{8}$ D. Mauri, H. C. Siegmann, P. S. Bagus, and E. Kay, J. Appl. Phys. 62, 3047 (1987).

${ }^{9}$ N. C. Koon, Phys. Rev. Lett. 78, 4865 (1997)

${ }^{10}$ T. C. Schulthess and W. H. Butler, J. Appl. Phys. 85, 5510 (1999).

${ }^{11}$ A. P. Malozemoff, Phys. Rev. B 35, 3679 (1987).

${ }^{12}$ A. P. Malozemoff, J. Appl. Phys. 63, 3874 (1988).

${ }^{13}$ A. P. Malozemoff, Phys. Rev. B 37, 7673 (1988).

${ }^{14}$ J. Spray and U. Nowak, J. Phys. D 39, 4536 (2006).

${ }^{15}$ P. Miltényi, M. Gierlings, J. Keller, B. Beschoten, G. Güntherodt, U. Nowak, and K. D. Usadel, Phys. Rev. Lett. 84 4224 (2000).

${ }^{16}$ U. Nowak, A. Misra, and K. D. Usadel, J. Appl. Phys. 89, 7269 (2001).

${ }^{17}$ J. Keller, P. Miltényi, B. Beschoten, G. Güntherodt, U. Nowak, and K. D. Usadel, Phys. Rev. B 66, 014431 (2002).

${ }^{18}$ U. Nowak, K. D. Usadel, J. Keller, P. Miltényi, B. Beschoten, and G. Güntherodt, Phys. Rev. B 66, 014430 (2002).

${ }^{19}$ T. Mewes, R. Lopusnik, J. Fassbender, B. Hillebrands, M. Jung, D. Engel, A. Ehresmann, and H. Schmoranzer, Appl. Phys. Lett. 76, 1057 (2000).
}

${ }^{20}$ A. Mougin, T. Mewes, M. Jung, D. Engel, A. Ehresmann, H. Schmoranzer, J. Fassbender, and B. Hillebrands, Phys. Rev. B 63, 060409(R) (2001)

${ }^{21}$ H. T. Shi, D. Lederman, and E. E. Fullerton, J. Appl. Phys. 91, 7763 (2002)

${ }^{22} \mathrm{~F}$. Nolting et al., Nature (London) 405, 767 (2000).

${ }^{23}$ H. Ohldag, A. Scholl, F. Nolting, S. Anders, F. U. Hillebrecht, and J. Stöhr, Phys. Rev. Lett. 86, 2878 (2001).

${ }^{24}$ D. Paccard, C. Schlenker, O. Massenet, R. Montmory, and A. Yelon, Phys. Status Solidi 16, 301 (1966).

${ }^{25}$ S. G. E. te Velthuis, A. Berger, G. P. Felcher, B. K. Hill, and E. D. Dahlberg, J. Appl. Phys. 87, 5046 (2000).

${ }^{26}$ A. F. Khapikov, J. W. Harrel, H. Fujiwara, and C. Hou, J. Appl. Phys. 87, 4954 (2000).

${ }^{27}$ K. Zhang, T. Zhao, and H. Fujiwara, J. Appl. Phys. 89, 6910 (2001).

${ }^{28}$ K. Zhang, T. Zhao, and H. Fujiwara, J. Appl. Phys. 91, 6902 (2002).

${ }^{29}$ C. Schlenker, S. S. P. Parkin, J. C. Scott, and K. Howard, J. Magn. Magn. Mater. 54-57, 801 (1986).

${ }^{30}$ T. Hauet, J. A. Borchers, P. Mangin, Y. Henry, and S. Mangin, Phys. Rev. Lett. 96, 067207 (2006).

${ }^{31}$ L. E. Fernandez-Outon, G. Vallejo-Fernandez, S. Manzoor, and K. O'Grady, J. Magn. Magn. Mater. 303, 296 (2006).

${ }^{32} \mathrm{U}$. Nowak, in Annual Reviews of Computational Physics IX, edited by D. Stauffer (World Scientific, Singapore, 2001), p. 105.

${ }^{33}$ U. Nowak and K. D. Usadel, Phys. Rev. B 44, 7426 (1991).

${ }^{34}$ B. Beckmann, U. Nowak, and K. D. Usadel, Phys. Rev. Lett. 91, 187201 (2003).

${ }^{35}$ B. Beckmann, K. D. Usadel, and U. Nowak, Phys. Rev. B 74, 054431 (2006). 
${ }^{36}$ M. Fecioru-Morariu, J. Wrona, C. Papusoi, and G. Güntherodt, Phys. Rev. B 77, 054441 (2008).

${ }^{37} \mathrm{~F}$. Radu and H. Zabel, in Magnetic Heterostructures: Advances and Perspectives in Spinstructures and Spintransport, edited by H. Zabel and S. Bader (Springer, Berlin, 2008), p. 97.

${ }^{38}$ S. Roy et al., Phys. Rev. Lett. 95, 047201 (2005).

${ }^{39}$ M. R. Fitzsimmons, B. J. Kirby, S. Roy, Z.-P. Li, I. V. Roshchin,
S. K. Sinha, and I. K. Schuller, Phys. Rev. B 75, 214412 (2007). ${ }^{40}$ R. Morales, Z.-P. Li, J. Olamit, K. Liu, J. M. Alameda, and I. K. Schuller, Phys. Rev. Lett. 102, 097201 (2009).

${ }^{41}$ F. Radu, M. Etzkorn, T. Schmitte, R. Siebrecht, A. Schreyer, K. Westerholt, and H. Zabel, J. Magn. Magn. Mater. 240, 251 (2002). 